\title{
Refractive-index profile measurement of preform rods by a transverse differential interferogram
}

\author{
Yasuo Kokubun and Kenichi lga
}

\begin{abstract}
A nondestructive refractive-index profile measurement of optical-fiber preform rods by means of a transverse-differential-interference method is given. The error resulting from the data reading of a fringe center is investigated by computer simulation and is confirmed to be less than about $1 \%$ of the index difference between the core and the cladding. The refractive-index profile of a preform rod was measured, and it almost coincided with that of the fiber drawn from the rod. The elliptic deformation of the core and its variation along the longitudinal axis were measured.
\end{abstract}

\section{Introduction}

Refractive-index profile measurement of optical-fiber preform rods is required in testing and sorting wellcontrolled graded-index multimode fibers and also in determining the cutoff frequency of single-mode fibers. ${ }^{1-3}$ To measure the refractive-index profile of preform rods, methods applied to optical fibers such as the longitudinal-interference method, ${ }^{4}$ the near-fieldpattern method, ${ }^{5}$ and the reflection method $^{6}$ seem to be applicable. However, since the preform rod is drawn into a fiber, its index profile must be measured nondestructively. The transverse-interference (TI) method ${ }^{7,8}$ can satisfy this demand with good accuracy. However, in applying this method to preform rods, the fringe shift becomes very large, thus overflowing from the interference-microscope field. An improvement in data collection $^{9}$ was made in order to overcome this difficulty, but in this method the resolution was limited because the amount of data in sampling the fringe shift is fixed.

In this paper, we show that the transverse-differential-interference (TDI) method ${ }^{10}$ that has been used for plastic focusing fibers can be applied to the measurement of the refractive-index profile of preform rods nondestructively. The accuracy of this method is investigated and compared with that of the transverseinterference (TI) method. The measuring technique

The authors are with Tokyo Institute of Technology, Research Laboratory of Precision Machinery \& Electronics, 4259 Nagatsuta, Midoriku, Yokohama, 227 Japan.

Received 1 June 1979.

0003-6935/80/060846-06\$00.50/0.

(c) 1980 Optical Society of America. and the index determination from the collected data are discussed.

\section{Experimental Setup and Index Determination}

The experimental setup is shown schematically in Fig. 1. The preform rod to be measured is immersed in index-matching oil and observed in its transverse direction with an interference microscope. The probing beam that has traversed the sample is divided into two arms by a Mach-Zehnder interferometer. Thus the divided beam $W_{2}$ is shifted an arbitrary distance $s$ by using a shearing prism. In the case of a drawn fiber, if the shearing distance is larger than the core radius, an ordinary TI pattern is observed. In the case of preform rods, however, it is impossible to observe the whole fringe shift because the optical path-length difference is much larger than the wavelength, and the fringe shift overflows from the microscope field.

On the other hand, if the shearing distance is small enough compared to the core radius, the fringe shift represents approximately the differential of the optical path-length difference between two paths, namely, one passing through the core and the other through the cladding. This is the basis of the TDI method for the measurement of a preform rod with a large core diameter.

Although this optical path-length difference is much larger than the wavelength, it is possible to make the fringe shift approximately small by regulating the shearing distance. A high-radiance GaAs LED with a small spectrum width $(\lambda=0.867 \mu \mathrm{m}, \Delta \lambda=0.027 \mu \mathrm{m})$ was used to obtain sharp fringes. The differential interference pattern is observed by means of an IR TV camera, and the refractive-index profile is obtained by calculating the fringe shift data. by $^{7}$ 


$$
n(u)=n_{2} \times \exp \left(-\frac{1}{\pi} \int_{0}^{\pi / 2} \frac{\lambda}{D s} R_{d}(y) \frac{\left[a^{2}-\left(\frac{u}{n_{2}}\right)^{2}\right]^{1 / 2} \cos \theta}{n_{2}\left\{\left(\frac{u}{n_{2}}\right)^{2}+\left[a^{2}-\left(\frac{u}{n_{2}}\right)^{2}\right] \sin ^{2} \theta\right\}^{1 / 2}} d \theta\right),
$$

$$
\begin{aligned}
y & =\left\{\left(\frac{u}{n_{2}}\right)^{2}+\left[a^{2}-\left(\frac{u}{n_{2}}\right)^{2}\right] \sin ^{2} \theta\right\}^{1 / 2}, \\
r & =u /[n(u)],
\end{aligned}
$$

where $n_{2}$ is the refractive index of the cladding, $a$ is the core radius, $D$ is the distance between fringes corresponding to one wavelength, $s$ is the shearing distance, $\lambda$ is the wavelength, and $R_{d}(y)$ is the fringe shift. The refraction of the probing ray is taken into account. The principal error of this formula is within $0.1 \%$.

\section{Evaluation of Reading Error}

The effect of the reading error generated in determining the fringe center is investigated by computer simulation. When the difference between indices initially given and calculated is designated $\delta n$, its standard deviation is defined by

$$
\epsilon=\left(\left\langle\delta n^{2}\right\rangle-\langle\delta n\rangle^{2}\right)^{1 / 2}
$$

Now we designate the reading error $\delta R$. A computer simulation was performed to make $\delta R$ clear, as follows: (1) The ideal fringe shift of the $\alpha$ power-law index profile was calculated numerically. (2) The reading error was assumed to be governed by the Gaussian distribution, and the quasi-Gaussian random number of the standard deviation of $\delta R$ was added to the initially given fringe shift. (3) The refractive-index profile was calculated from this fringe shift, including quasiGaussian random noise, with the aid of Eqs. (1)-(3). (4) The standard deviation $\epsilon$ was calculated with the aid of Eq. (4), and $\epsilon$ was compared with $\delta R$ for the various values of $\alpha$. From this computer simulation, the relation between $\delta R$ and $\epsilon$ for the TDI method was obtained as follows:

$$
\frac{\epsilon}{\Delta n}=K_{d} \frac{\lambda}{2 s \Delta n} \frac{\delta R}{D}
$$

where $K_{d}$ was the constant number independent of the index profile and the sampling number, $K_{d}=0.19$.
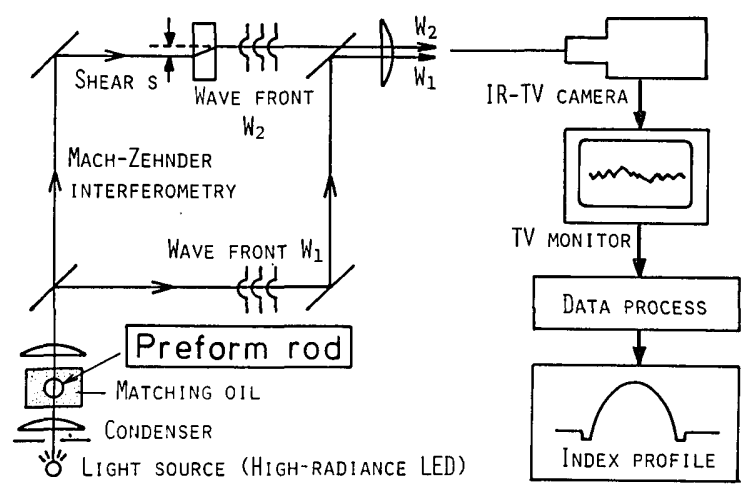

Fig. 1. Measuring system of transverse differential interferometry.

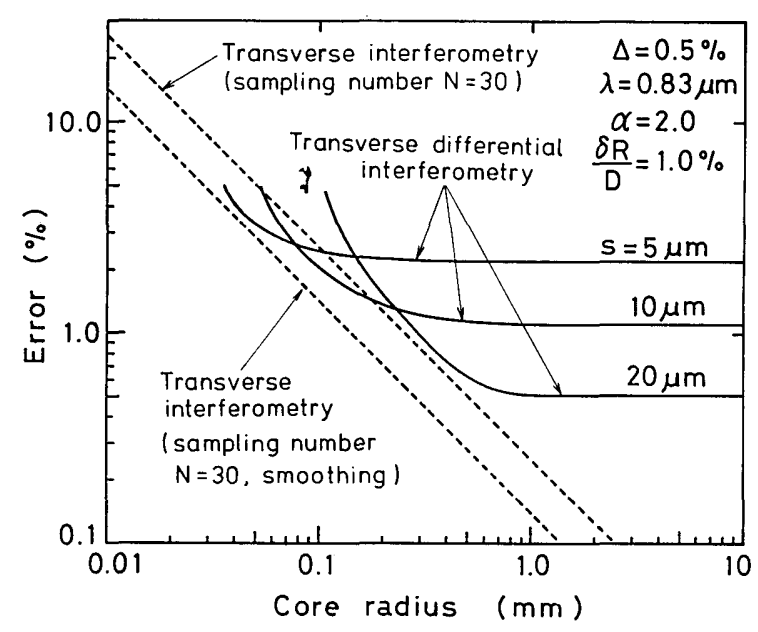

Fig. 2. Core-radius dependence of the error resulting from the data reading.

In the same way, the relation between $\Delta R$ and $\epsilon$ for the TI method, which has been applied to optical fibers, was obtained as follows:

$$
\frac{\epsilon}{\Delta n}=K_{t} \frac{\lambda}{2 a \Delta n} \frac{\delta R}{D}
$$

where $K_{t}$ was the coefficient independent of the index profile and was expressed in terms of the sampling number $N$ as

$$
K_{t}=0.15(N-1) .
$$

For example, in the case of $\Delta=0.5 \%, \lambda=0.83 \mu \mathrm{m}$, and $\delta R / D=1.0 \%$, the error resulting from the data reading vs core radius is evaluated as shown in Fig. 2. In the case of the TI method, the error decreases with the increase in the core radius. On the other hand, the fringe shift becomes larger with the increase in the core radius, and it finally overflows from the field of the microscope. Therefore, the TI method seems to be limited to $\lesssim 500 \mu \mathrm{m}$ of the core radius.

In the case of the TDI method, however, the error resulting from the data reading is independent of the core radius as long as the shearing distance is small enough compared to the core radius. When the core radius becomes less than about ten times the shearing distance, the fringe shift cannot be approximated by the differential of the optical path-length difference between two paths passing through the core and the cladding, respectively. Therefore, the error resulting from the above-mentioned fact is larger than that from the data reading. The critical core radius, where the former error becomes larger than the latter, does not critically depend upon the index profile. Those errors are shown in Fig. 2 with an $\alpha$ value of 2 . If the preform rod of a $>1-\mathrm{mm}$ core radius is measured with the 


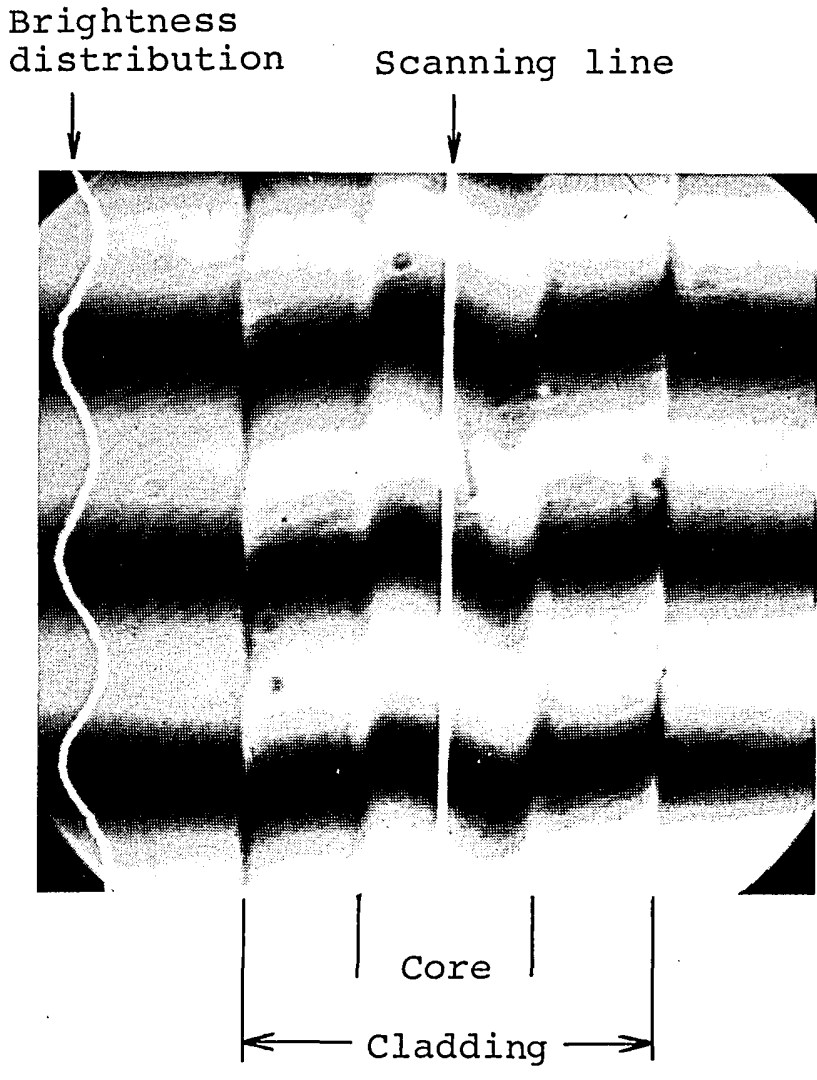

Fig. 3. TV monitor indicating brightness distribution. (The sample of this figure is a graded-index multimode fiber.) shearing distance of $20 \mu \mathrm{m}$, the total error is about half of the reading error. Therefore, the TDI method is suitable for measurement of preform rods whose core radius is $>1 \mathrm{~mm}$.

\section{Measurement}

The transverse differential fringe shift was obtained as follows: The TDI pattern is observed by means of an IR TV camera. The TV monitor displays the brightness distribution along a scanning line as shown in Fig. 3. The fringe shift was easily obtained by reading the minimum point of the brightness distribution. The measuring data were obtained by shifting the sample and by collecting the fringe shift corresponding to each point. The refractive index was calculated from these data with the aid of Eqs. (1)-(3).

Some reduction of the measuring time and the reading error seems possible by connecting the IR TV and minicomputer directly. This automated apparatus is being prepared.

Figure 4 shows the TDI pattern of a $\mathrm{GeO}_{2}$-doped preform rod for a graded-index multimode fiber fabricated by the CVD method. Small ripples of the fringe shift were due to the index fluctuation in each deposited layer. The number of layers was about seventy. In order to determine the whole index profile, it is necessary to obtain the smoothed envelope of this differential interference fringe. Therefore, as shown in Fig. 5, the mean values of the fringe shifts and the transverse distances of the two adjacent points were used as the sampled data of the smoothed envelope. From these

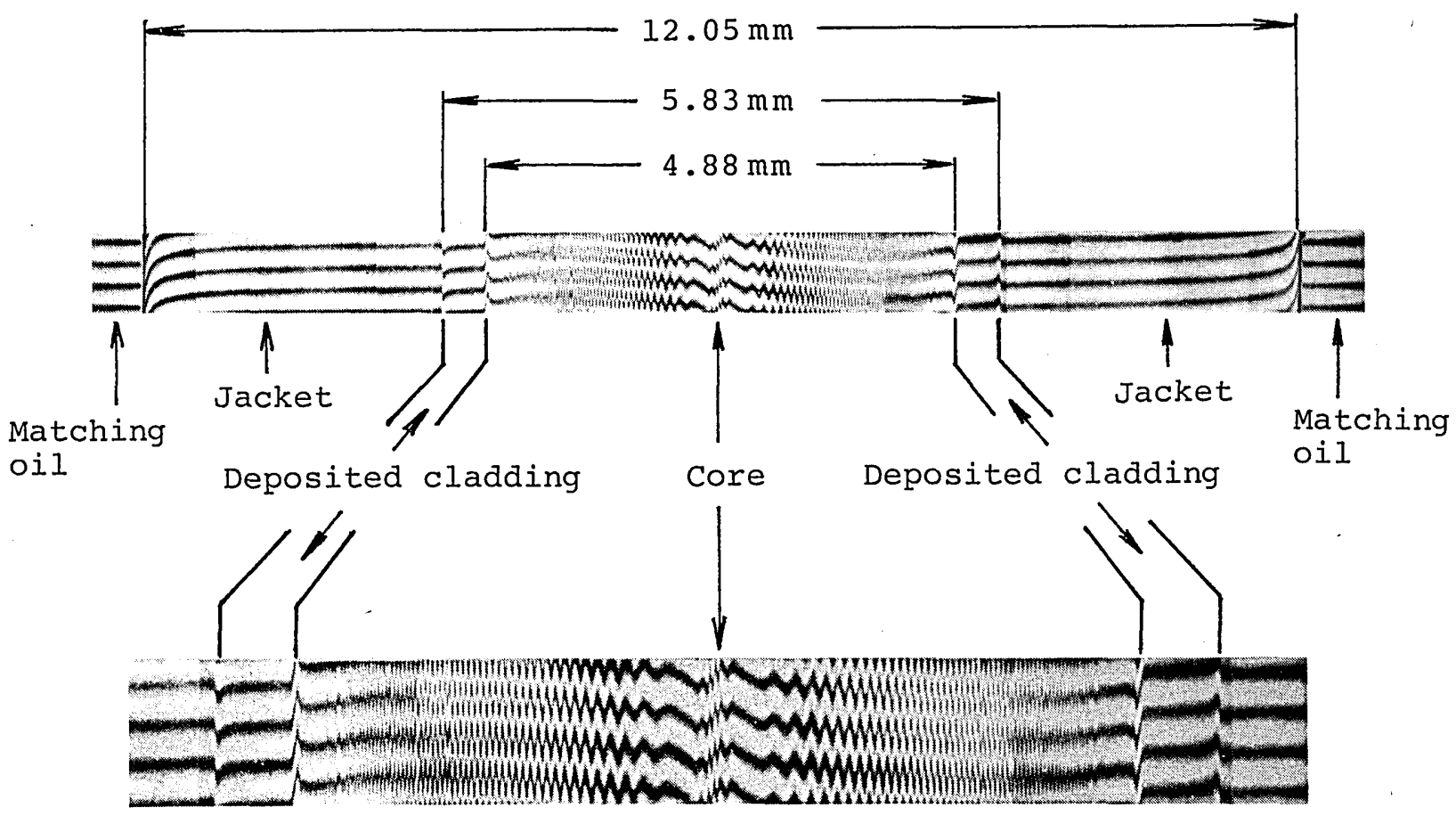

Fig. 4. Transverse-differential-interference pattern of a preform rod with $\mathrm{GeO}_{2}$-doped core fabricated by the CVD method. 


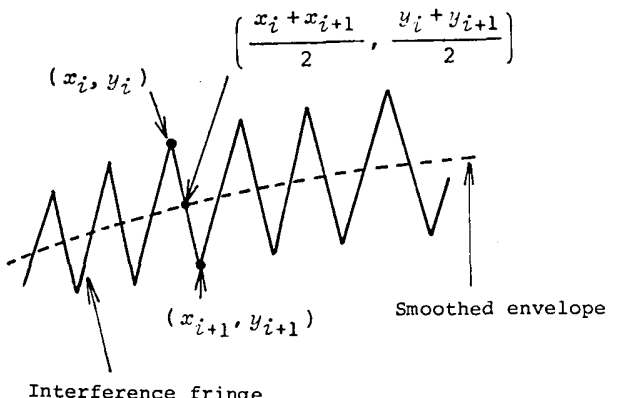

Fig. 5. Smoothing of ripples in the differential interference fringe.

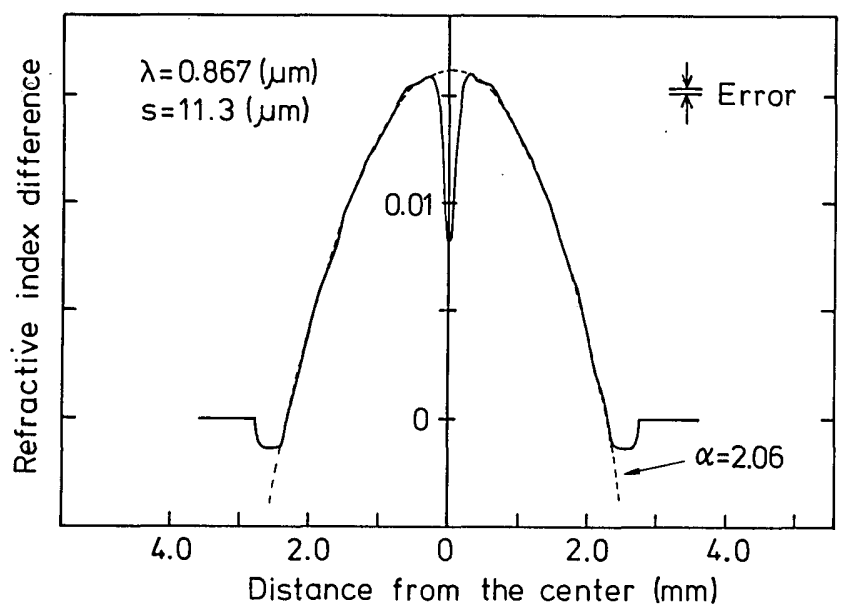

Fig. 6. Refractive-index profile of a preform rod for a graded-index multimode fiber.

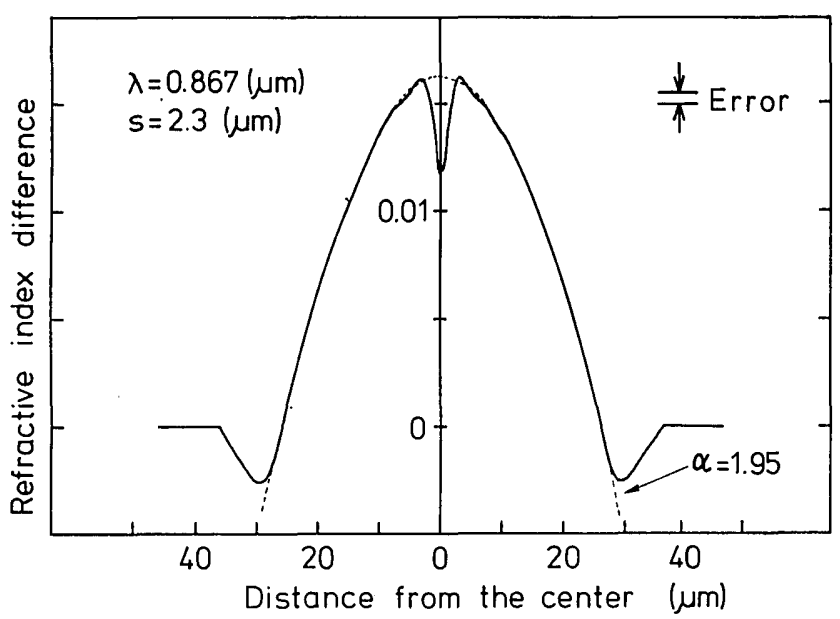

Fig. 7. Refractive-index profile of a fiber drawn from the preform rod of Fig. 6.

data, the refractive-index profile was obtained. The result is shown in Fig. 6. The least-squares fit of $\alpha$ is 2.06. The error resulting from the data reading was evaluated with the aid of Eq. (7) to be \pm 0.0002 (1.3\% of the index difference between the core and the cladding).

The refractive-index profile of the fiber drawn from this perform rod was also measured by means of the TDI method. The result is shown in Fig. 7. The error re- sulting from the data reading in this measurement was evaluated to be \pm 0.0005 . Figure 8 shows the comparison of the refractive-index profile of the preform rod with that of the fiber. The abscissa is the distance from the center normalized by the core radius. These profiles are almost similar. Figure 9 shows the refractive-index profile of the fiber drawn from the same rod, measured by the NFP method. The least-squares fit of $\alpha$ calculated from the profiles shown in Figs. 7 and 9 are 1.95 and 2.05 , respectively. These values almost coincide with that of the preform rod.

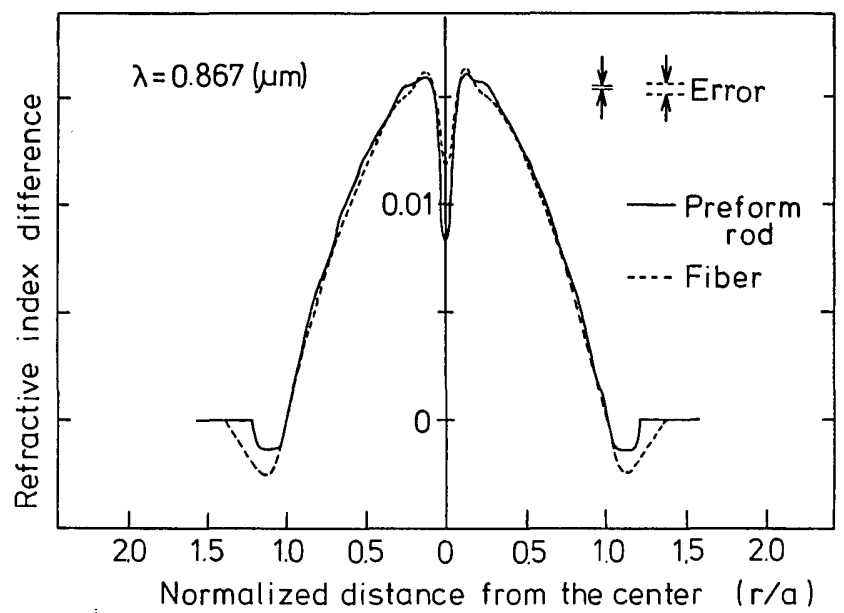

Fig. 8. Comparison of index profiles of the preform rod (solid curve) and of the fiber drawn for the rod (dashed curve).

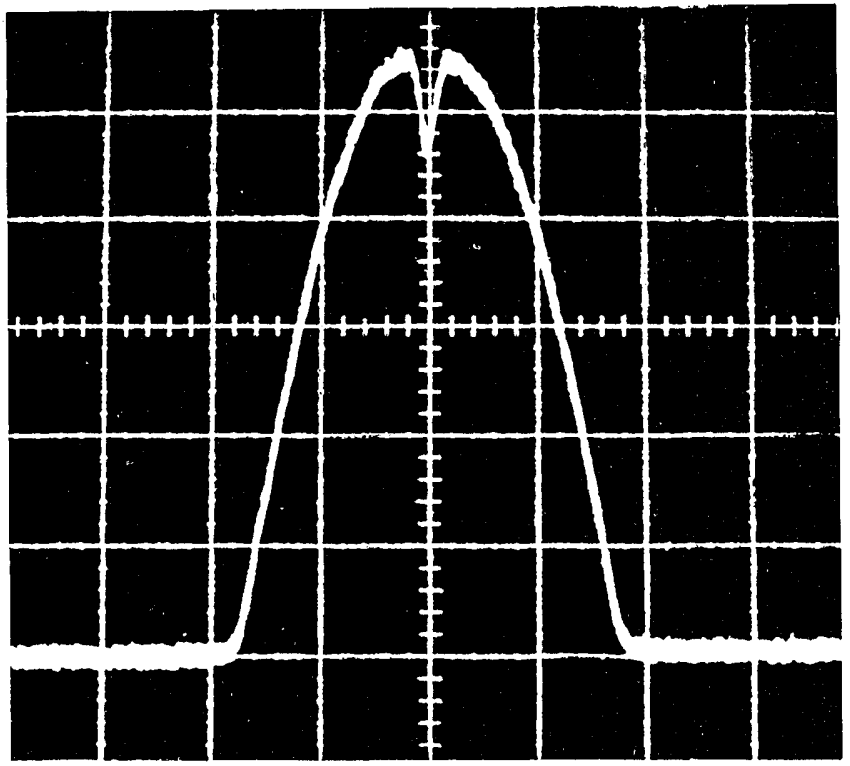

Fig. 9. Refractive-index profile of a fiber drawn from the same preform rod, measured by the NFP method. 


\section{Elliptic Deformation}

The elliptic deformation of the core was observed by rotating the preform rod. The core center was fortunately determined by observing the sharp ripple in the differential interference pattern due to the center dip of the index profile as shown in Fig. 4. The corecladding boundary was determined in the same way. So that the radius and the diameter of the core could be determined separately, the core radius and diameter were measured by shifting the sample and by measuring the shifted distance by means of a microgauge. Figure 10 shows the change of the diameters and the core radii from different sides and of the deposited cladding. The azimuth angle of $0 \mathrm{rad}$ shows the direction in which the index profile shown in Fig. 6 was measured. Since the diameter changes similarly to the radius, it can be concluded that this preform rod has elliptic deformation. The ratio of the major and the minor axes was about 0.975 .

The core-diameter variations of the major and the minor axes along the longitudinal axis are shown in Fig. 11. The elliptic deformation shown in Fig. 10 was measured at point $C$. In the region $>4 \mathrm{~cm}$ away from the end (point $E$ ), the variation was almost within $0.6 \%$. Furthermore, the ratio of diameters of the major and the minor axes was almost constant along the longitudinal axis. Figure 12 shows the directions of the major and the minor axes vs the longitudinal distance. The azimuth angle fluctuation along the longitudinal axis is within $\pi / 12 \mathrm{rad}$, and the rotation of the major and minor axes was not observed. From these facts, it can be concluded that the elliptic deformation of the core is almost uniform along the longitudinal axis.

\section{Conclusions}

The TDI method was applied to determine nondestructively the index profile of preform rods. The accuracy of this method was investigated by computer

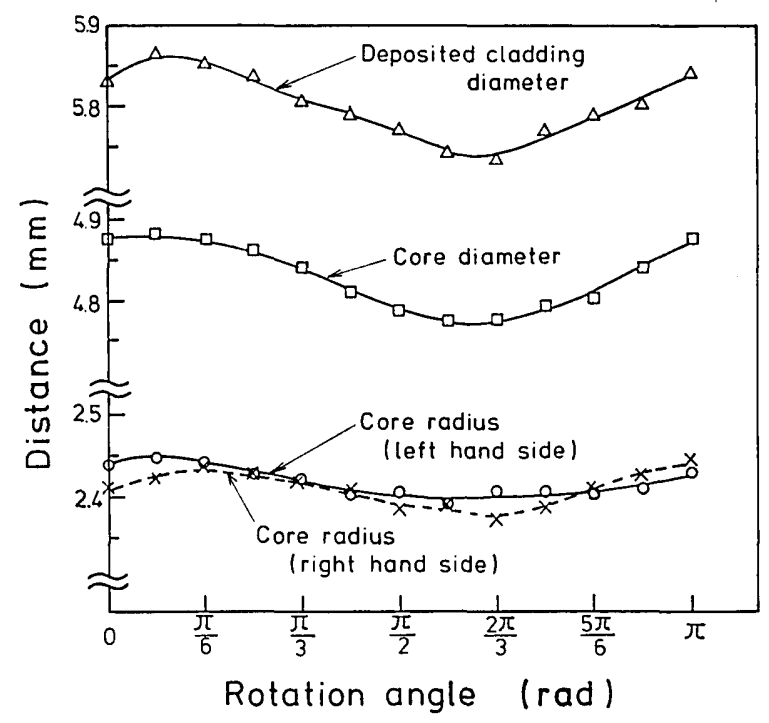

Fig. 10. Core-diameter and radius variation vs rotation angle.

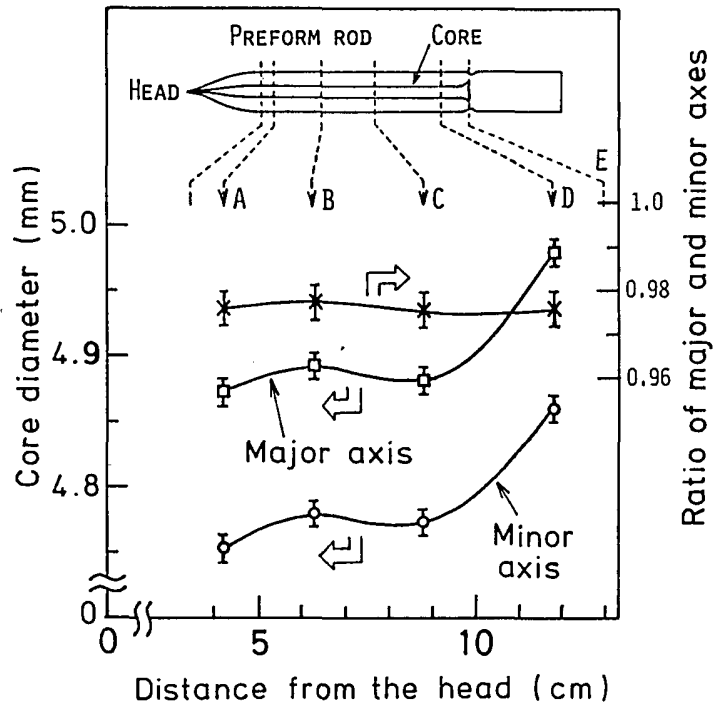

Fig. 11. Longitudinal variations of the core diameters of the major and the minor axes.

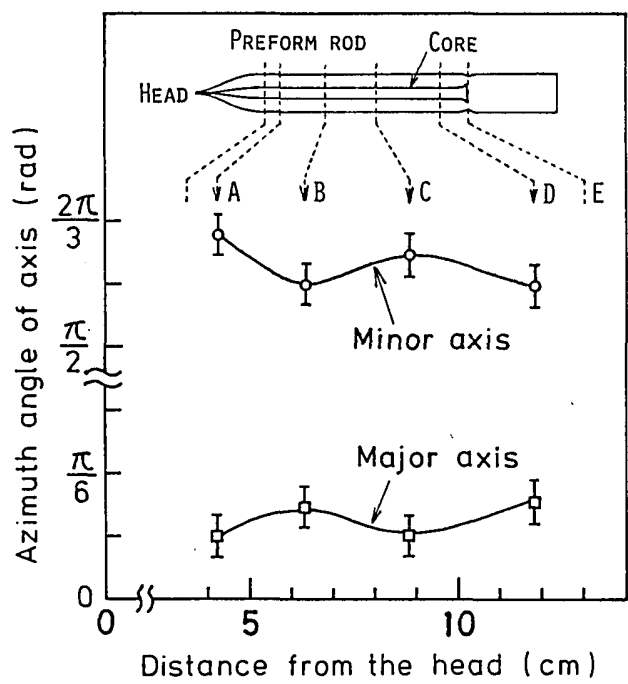

Fig. 12. Longitudinal variations of directions of the major and the minor axes.

simulations. The formula for evaluating the error resulting from the data reading of the fringe center was derived, and it was confirmed that the error resulting from the data reading is independent of the core radius. If the reading error of the fringe center $\delta R / D$ is reduced $<2 \%$, the total measuring error can be reduced to within $1 \%$ of the index difference between the core and the cladding. The index profiles of a preform rod and the fiber drawn from the rod were measured, and it was confirmed that these profiles are similar. In addition, the elliptic deformation of the core was measured, and it was confirmed that the elliptic deformation was almost uniform along the longitudinal axis. 
The authors express their sincere thanks to $\mathrm{Y}$. Suematsu and K. Furuya for their valuable discussions of this work and to T. Omoto and M. Oikawa for their help in the experiments. They also wish to thank S. Tanaka and K. Inada of Fujikura Cable Works for supplying the preform rods and K. Kurata of the Central Research Laboratory, Hitachi, Limited, for supplying the high-radiance LED.

\section{References}

1. Y. Kokubun and K. Iga, Paper of TGOQE, Institute of Electronics and Communication Engineers Japan, No. OQE78-99 (1978).

2. K. Hotate and T. Okoshi, Trans. Inst. Electron. Commun. Eng. Jpn. E62, No. 1, 1 (1979).
3. T. Tanaka and Y. Suematsu, Trans. Inst. Electron. Commun. Eng. Jpn. E59, No. 11, 1 (1976).

4. W. E. Martin, Appl. Opt. 13, 2112 (1974).

5. T. Miya, M. Horiguchi, K. Senda, and T. Edahiro, National Convention Records of Institute of Electronics and Communications Engineers Japan, No. 818 (1978).

6. M. Ikeda, M. Tateda, and H. Yoshikiyo, Appl. Opt. 14, 814 (1975).

7. Y. Kokubun and K. Iga, Trans. Inst. Electron. Commun. Eng. Jpn. E60, No. 12, 702 (1977).

8. Y. Kokubun and K. Iga, Trans. Inst. Electron Commun. Eng. Jpn. E61, No. 3, 184 (1978).

9. T. Okoshi and N. Takada, Paper of TGOQE, Institute of Electronics and Communication Engineers Japan, No. OQE78-62 (1978).

10. K. Iga and N. Yamamoto, Appl. Opt. 16, 1305 (1977).

\section{Books continued from page 845}

then current level of research. The main subtopics were presented by 14 mostly very well established authorities with 6 presentations on more specific topics and 4 additional short presentations including one on the proper usage of the Greek alphabet in scientific publications.

The treatment is thorough, covering nearly 700 well-presented printed pages. Individual lectures include an introductory overview, in which some pure (hot luminescence and electron-hold condensation) and applied topics (electroluminescent cells) are highlighted. Then follows more extended presentations on interaction of light with ions in solids; nonradiative, multiphonon decay; optical properties of transition metal ions in solids (theory and experiment including concentration quenching); optical properties of rare-earth ions; luminescence of main-group (closed-shell) ions; properties of pure ionic crystals (collective excitations, phonons, excitons, and magnons); optical properties of the more covalent semiconducting materials (direct and indirect gaps, bound and free excitons, various types of luminescence activator); energy states of strongly interacting ions; polaron effects; effects of hydrostatic pressure; and materials science of inorganic luminescent solids (activator-host interactions, quantum efficiency, energy transport, concentration quenching). The latter chapter leads to general rules for the design of efficient photon and cathode ray-excited phosphors. Then follow chapters on hot luminescence and light scattering; and applications and recent techniques including coherent, fast pulse, and photoacoustic spectroscopy. The medium-length specialized presentations cover the behavior of concentrated $\mathrm{Mn}^{2+}$ systems, optical properties of rare gas solids, and reorientation in molecular centers.

This book will reward study by all interested in the subject, particularly those at the postgraduate level. The principal drawback is the disproportionally small attention given to luminescence of semiconducting materials, well under $10 \%$ of the total page count.

\section{P. J. DEAN}

Neutron Diffraction. Edited by H. DACHS. Springer-Verlag, New York, 1978. 357 pp. $\$ 29.90$.

The purpose of this monograph is to discuss the technical aspects of neutron diffraction to help researchers decide whether neutron diffraction would be useful in their work. Thus it stresses the areas in which neutron diffraction makes a unique contribution to understanding but is not a cookbook of how to carry out neutron diffraction work. The contributors to the book discuss their specialties with insight and clarity. Consequently the book admirably achieves its goal.

There are nine chapters. After an introduction dealing with the principles of neutron diffraction there are chapters written by experts who discuss their special areas of research. The topics covered are neutron diffraction's contribution to the study of hydrogen bonding, isotope effects, and molecular biology; the determination of magnetic structures and disordered structures; phase transitions; critical phenomena; the structure of liquids; and crystal perfection.

The book has a distinctly experimental flavor. For example, there is a chapter devoted to polarized neutrons that discusses how to make polarized beams and what one might investigate with them. In addition, although many equations appear throughout, nowhere in the book does an author get bogged down in a long derivation-a nice feature. The book immediately gets down to results and is very readable. Hence, much can be learned with only a small amount of time and effort.

Only one of the ten authors is English or American. Nevertheless, all the chapters are written in good idiomatic English. There are some quaint usages, such as "ambition" for goal, but these are few and cause no misunderstanding. The authors and the editor have done an outstanding job in this regard. Also, the editor has coordinated the articles of the various authors with a fine hand. They are all written at nearly the same level of technicality, and they fit together well, producing a coherent unit.

This book gives an excellent up-to-date overview of applications of neutron diffraction. It should be a part of the library of all colleges and universities.

\section{ALBERT C. CLAUS}

Advances in Image Pickup and Display, Vol. 3. Edited by B. KAZAN. Academic Press, New York, 1977. 302 pp. $\$ 31.00$.

This third volume edited by Ben Kazan consists of three short monographs. The first, by $\mathbf{B}$. Singer, is a concise well-written article beginning with a brief resume running through ferroelectric materials and the generation of pyroelectric signals. Those more deeply interested should look into PRINCIPLES AND APPLICATIONS OF FERROELECTRICS AND RELATED MATERIALS by M. E. Lines and A. M. Glass (Clarendon, Oxford, 1977).

Singer quickly proceeds to a derivation of pyroelectric signals in a manner that differs from previous analyses of Putley, Garn, Sharp, and Park. To his credit, Singer points out, in Eq. (6), how some characteristics can lead to specific undesirable artifacts in pyroelectric cameras. The details of the performance of such cameras, the fabrication of the pyroelectric heart of the tube, and the various modes of operation are well described from both a basic theoretical point of view and a reasonable view of the practical.

My only serious disappointment with this otherwise excellent article by Singer needs few words. In contrast to the rugged $\mathrm{Sb}_{2} \mathrm{~S}_{3}$ or $\mathrm{Si}$ vidicons, the pyroelectric element is stretched like a drumhead across a hoop. It vibrates like a drumhead undamped in the vacuum of the tube. Singer does not warn the reader that the microphonics can be overwhelming compared with the Johnson noise of Singer's formal analysis. The potential user should investigate carefully.

continued on page 862 